\title{
ON TOPOLOGICAL PROPERTIES OF PLANE GRAPHS BY USING LINE OPERATOR ON THEIR SUBDIVISIONS
}

\author{
Xu $\mathrm{Li}^{1}$, Fatima Asif ${ }^{2}$, Zohaib Zahid ${ }^{2}$, Sohail Zafar ${ }^{2} \S$ \\ ${ }^{1}$ Sino-German Institute of Applied Mathematics \\ Hefei University, Hefei 230601, CHINA \\ 2 Department of Mathematics \\ University of Management and Technology (UMT) \\ Lahore, PAKISTAN
}

\begin{abstract}
In this paper, we will compute some topological indices such as Zagreb indices $M_{1}(G), M_{2}(G), M_{3}(G)$, Zagreb coindices $M_{1}(\bar{G}), \overline{M_{1}}(G)$, $\left.M_{2}(\bar{G}), \overline{M_{2}}(G)\right), \overline{M_{2}}(\bar{G})$, hyper-Zagreb index $H M(G)$, atom-bond connectivity index $A B C(G)$, sum connectivity index $\chi(G)$, augumented Zagreb index $A Z I$ and geometric-arithmetic connectivity index $G A(G)$ of line graph of subdivision of some plane graphs.
\end{abstract}

AMS Subject Classification: 05C07, 05C35, 05C40

Key Words: topological indices, line graph, convex polytopes

\section{Introduction}

Topological indices are real numbers that are presented as graph parameters (e.g. the degree of vertices, distances, etc.) introduced during studies conducted on the molecular graphs in chemistry and can describe some physical and chemical properties of molecules. Topological indices are numerical parameters of a graph which are invariant under graph isomorphisms.

Let $G$ be a connected graph with vertex set $V(G)$ and edge set $E(G) \subseteq$ $V(G) \times V(G)$. Let $p=|V(G)|$, the order of $G$ and $q=|E(G)|$, the size of $G$.

Received: June 17, 2019

(C) 2019 Academic Publications

${ }^{\S}$ Correspondence author 
The complement of a graph $G$, denoted by $\bar{G}$, is a simple graph having same set of vertices $V(G)$ in which any two vertices that are connected by an edge, if and only if they are not adjacent in $G$. Consequently, $E(G) \cup E(\bar{G})=E\left(K_{p}\right)$ and we obtain a complete graph $K_{p}$ of order $p$, and $|E(\bar{G})|=\frac{p(p-1)}{2}-q$. The degree $d_{v}$ of any vertex $v$ is defined as the number of vertices joining to that vertex $v$ and the degree $d_{e}$ of an edge $e \in E(G)$ is defined as the number of its adjacent vertices in $V(L(G)$ ), where $L(G)$ is the line graph whose vertices are the edges of $G$, with $u v \in E(L(G)$ when $u$ and $v$ have a common end point in $G$. In structural chemistry, line graph of a graph $G$ is very useful. The first topological indices on the basis of line graph was introduced by Bertz in 1981 (see [4]). For more details on line graph (see [8, 16, 17, 18, 20, 22, 25]). The subdivision $S(G)$ of a graph $G$ can be obtained by replacing each edge of $G$ by a path of length 2. Further details on the topological indices of $L(S(G))$ can be viewed in the articles (see $[3,9,10,21,23,24]$ ). From this motivational work, we calculate the topological indices of line graph of subdivision of Jahangir graph, Banana Tree graph and Firecracker graph.

\section{Some Degree-Based Topological Indices}

Analyzing the structure-dependency of total $\pi$-electron energy, first Zagreb index $M_{1}(G)$ and second Zagreb index $M_{2}(G)$ were introduced (see [17]). They are defined as follows:

$$
\begin{aligned}
& M_{1}(G)=\sum_{u \in V(G)} d_{u}^{2} \\
& M_{2}(G)=\sum_{u v \in E(G)} d_{u} d_{v} .
\end{aligned}
$$

For more properties of the Zagreb indices (see [5, 13, 15, 19, 30]). In 1977, Alberton introduced the irregularity of graphs (see [1]). To confirm with the terminology of chemical graph theory Fath-Tabar (see [7]) called Alberton's irregularity the third Zagreb index and is defined as:

$$
M_{3}(G)=\sum_{u v \in E(G)}\left|d_{u}-d_{v}\right| .
$$

In 2013, Shirdel, RezaPour and Sayadi (see [27]) introduced the hyperZagreb index. It is defined as follows:

$$
H M(G)=\sum_{u v \in E(G)}\left(d_{u}+d_{v}\right)^{2} .
$$


Randic introduced the so-called Randic index in 1975 (see [26]). It is defined as follows:

$$
R(G)=\sum_{u v \in E(G)} \frac{1}{\sqrt{d_{u} d_{v}}} .
$$

Estrada et al. (see [6]) introduced a new topological index and named it atom-bond connectivity index, defined as follows:

$$
A B C(G)=\sum_{u v \in E(G)} \sqrt{\frac{d_{u}+d_{v}-2}{d_{u} d_{v}}} .
$$

The $A B C$ index is excellently correlated with the thermodynamic properties of alkanes.

Zhou and Trianjstic introduced the general sum-connectivity index $\chi(G)$ (see [29]). It is defined as follows:

$$
\chi(G)=\sum_{u v \in E(G)} \frac{1}{\sqrt{d_{u}+d_{v}}} .
$$

Vukicevic and Furtula defined the first geometric arithmetic index $G A$ (see [28]), defined as follows:

$$
G A(G)=\sum_{u v \in E(G)} \frac{2 \sqrt{d_{u} d_{v}}}{d_{u}+d_{v}} .
$$

Furtula et al. put forward the following modified version of the $A B C$ index and named it as Augumented Zagreb index $A Z I$ (see [11]). It is defined as follows:

$$
A Z I(G)=\sum_{u v \in E(G)}\left(\frac{d_{u} d_{v}}{d_{u}+d_{v}-2}\right)^{3} .
$$

The first Zagreb coindex is defined as (see [14]):

$$
\overline{M_{1}}=\overline{M_{1}}(G)=\sum_{u v \notin E(G)}\left[d_{u}+d_{v}\right] .
$$

The second Zagreb coindex is defined as:

$$
\overline{M_{2}}=\overline{M_{2}}(G)=\sum_{u v \notin E(G)} d_{u} d_{v} .
$$


Theorem 1. (see [12]) Let $G$ be a graph of order $p$ and size $q$. Then,

$$
\begin{aligned}
& M_{1}(\bar{G})=M_{1}(G)+p(p-1)^{2}-4 q(p-1) \\
& \overline{M_{1}}(G)=2 q(p-1)-M_{1}(G) \\
& \overline{M_{1}}(\bar{G})=2 q(p-1)-M_{1}(G) .
\end{aligned}
$$

Theorem 2. (see [14]) Let $G$ be a graph of order $p$ and size $q$. Then,

$$
\begin{gathered}
M_{2}(\bar{G})=\frac{1}{2}(p-1)^{2}\left(p^{2}-p-3 q\right)+2 q^{2}+\frac{2 p-3}{2} M_{1}(G)-M_{2}(G) ; \\
\overline{M_{2}}(G)=2 q^{2}-\frac{1}{2} M_{1}(G)-M_{2}(G) ; \\
\overline{M_{2}}(\bar{G})=q(p-1)^{2}-(p-1) M_{1}(G)+M_{2}(G) .
\end{gathered}
$$

\section{Topological indices of line graph of subdivision of the Jahangir graph}

Jahangir graph $J_{n, m}$ consisting of a cycle $C_{n m}$ with one additional vertex which is adjacent to $m$ vertices of $C_{n m}$ at distance $n$ to each other on $C_{n m}$ (see [2]). The subdivision of Jahangir graph has additional vertex between each pair of vertices. The subdivision of $J_{n, m}$ has order $m(2 n+1)$ and size $2 m(n+1)$ as shown in Fig. 1(b).

Theorem 3. Let $L\left(S\left(J_{n, m}\right)\right)$ be the line graph of subdivision of the Jahangir graph $J_{n, m}$. Then,

1. $M_{1}\left[L\left(S\left(J_{n, m}\right)\right)\right]=m^{2}(m-1)+4 m(2 n-3)+28 m+m(m+3)$;

2. $M_{2}\left[L\left(S\left(J_{n, m}\right)\right)\right]=\frac{1}{2} m^{3}(m-1)+4 m(2 n-3)+39 m+3 m^{2}$;

3. $M_{3}\left[L\left(S\left(J_{n, m}\right)\right)\right]=m^{2}-m$;

4. $H M\left[L\left(S\left(J_{n, m}\right)\right)\right]=16 m(2 n-3)+158 m+m(3+m)^{2}+2 m(m-1) m^{2}$;

5. $A B C\left[L\left(S\left(J_{n, m}\right)\right)\right]=\frac{1}{2} m(2 n-3) \sqrt{2}+m \sqrt{2}+2 m+\frac{1}{\sqrt{3}} m \sqrt{\frac{m+1}{m}}+\frac{1}{2}(m-$ 1) $\sqrt{2 m-2}$

6. $G A\left[L\left(S\left(J_{n, m}\right)\right)\right]=2 m n+\frac{12 m \sqrt{(m+6)}}{15+m}+\frac{2 m \sqrt{(m+6) m}}{2 m+6}+\frac{1}{2} m(m-1)$; 


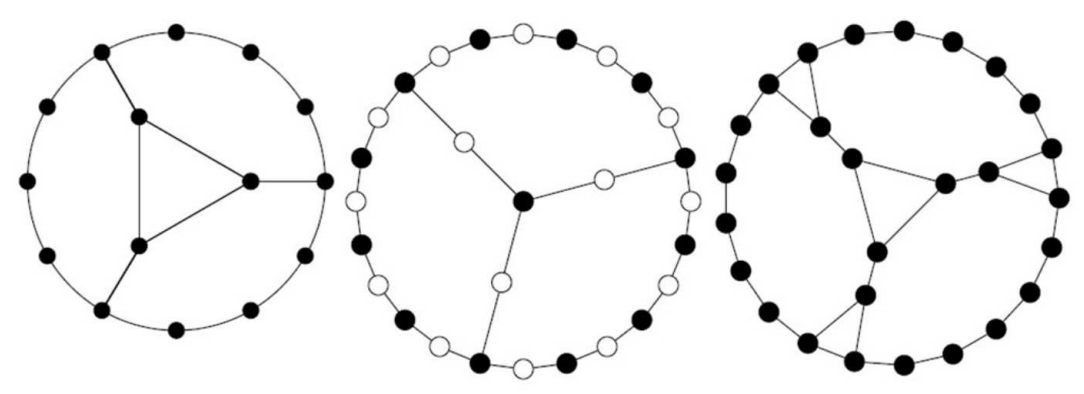

(a)

(b)

(c)

Figure 1: The Jahangir graph, subdivision and line graph of subdivision of Jahangir graph $J_{4,3}$.

\begin{tabular}{cccccc}
\hline$\left(d_{u}, d_{v}\right)$ where $u v \in E(G)$ & $(2,2)$ & $(2,3)$ & $(3,3)$ & $(3, m)$ & $(m, m)$ \\
\hline Number of edges & $m(2 n-3)$ & $2 m$ & $3 m$ & $m$ & $\frac{m(m-1)}{2}$ \\
\hline
\end{tabular}

Table 1: The edge partition of $L\left(S\left(J_{n, m}\right)\right)$.

7. $\chi\left[L\left(S\left(J_{n, m}\right)\right)\right]=\frac{1}{2} m(2 n-3)+\frac{2}{5} m \sqrt{5}+\frac{1}{2} m \sqrt{6}+\frac{m}{\sqrt{3+m}}+\frac{\sqrt{2}}{4} \sqrt{m}(m-1)$;

8. $A Z I\left[L\left(S\left(J_{n, m}\right)\right)\right]=8 m(2 n-3)+\frac{3211}{64} m+\frac{27 m^{4}}{(1+m)^{3}}+\frac{1}{2} \frac{m^{7}(m-1)}{(2 m-2)^{3}}$.

Proof. The graph $L\left(S\left(J_{n, m}\right)\right)$ for $n=4$ and $m=3$ is shown in Fig. 1(c). The order of $L\left(S\left(J_{n, m}\right)\right)$ is $2 m(n+1)+1$ out of which $2 m(n-1)$ vertices are of degree $2,3 m$ vertices are of degree 3 and $m$ vertices are of degree $m$. The size of $\left.L\left(S\left(J_{n, m}\right)\right)\right) \mid$ is $\frac{m^{2}+4 m n+5 m}{2}$. The edge partition of $L\left(S\left(J_{n, m}\right)\right)$ into the edges of type $\left(d_{u}, d_{v}\right)$ where $u v$ is an edge of $L\left(S\left(J_{n, m}\right)\right)$ is shown in Table 1.

We obtain the required results by using formulas (1) - (9).

Theorem 4. Let $L\left(S\left(J_{n, m}\right)\right)$ be the line graph of subdivision of the Jahangir graph $J_{n, m}$. Then,

1. $M_{1} \overline{\left[L\left(S\left(J_{n, m}\right)\right)\right]}=8 m^{3} n^{3}+24 m^{3} n^{2}+203 m^{3} n-24 m^{2} n^{2}+5 m^{3}-53 m^{2} n-$ 


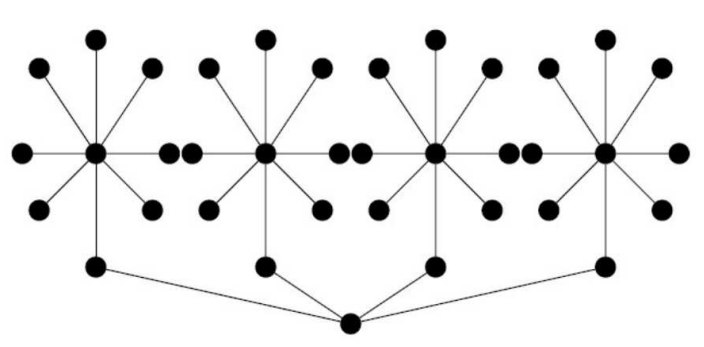

Figure 2: The Banana Tree graph $B_{7,4}$.

$269 m^{2}+18 m n+31 m ;$

2. $\overline{M_{1}}\left[L\left(S\left(J_{n, m}\right)\right)\right]=4 m^{3} n+16 m^{2} n^{2}+5 m^{3}+36 m^{2} n+18 m^{2}-16 m n+33 m$;

3. $M_{2} \overline{\left[L\left(S\left(J_{n, m}\right)\right)\right]}=m(n+1)(2 m(n+1)-1)^{3}-3\left(\frac{1}{2} m^{2}+2 m n+\frac{5}{2} m\right)(2 m(n+$ 1) -1$)^{2}-\frac{5}{2} m^{2}+2 m n-\frac{73}{2} m+8 m^{2}(n+1)(2 n-3)+28 m+m(m+3)+$ $m^{2}(m-1)-\frac{3}{2}-4 m(2 n-3)-\frac{1}{2} m^{3}(m-1) ;$

4. $\overline{M_{2}}\left[L\left(S\left(J_{n, m}\right)\right)\right]=\frac{1}{2}\left(m^{2}+4 m n+5 m\right)^{2}-6 m(2 n-3)-53 m-\frac{1}{2} m(m+$ 3) $-\frac{1}{2} m^{2}(m-1)-3 m^{2}-\frac{1}{2} m^{3}(m-1)$;

5. $\overline{M_{2}}\left(\overline{\left[L\left(S\left(J_{n, m}\right)\right)\right]}\right)=\frac{1}{2}\left(2 m^{2}(n+1)-1\right)^{2}+4 m n(2 m(n+1)-1)+5 m(2 m(n+$ 1) -1$)^{2}-8 m^{2}(n+1)(2 n-3)+28 m+m(3+m)+m^{2}(m-1)+1+4 m(2 n-$ $3)+39 m+3 m^{2}+\frac{1}{2} m^{3}(m-1)$.

Proof. It is easily seen from Theorems 1, 2 and Table 1.

\section{Topological indices of line graph of subdivision of Banana Tree graph}

The Banana tree graph $B_{n, m}$ is the graph obtained by connecting one leaf of each of $n$ copies of an $m$-star graph with a single root vertex that is distinct from all the stars. The subdivision of $B_{n, m}$ consist of an additional vertex between each pair of vertices, has order $2 m n+1$ and size $2 m n$ as shown in Fig. 3.

Theorem 5. Let $L\left(S\left(B_{n, m}\right)\right)$ be the line graph of subdivision of Banana tree graph $B_{n, m}$. Then 


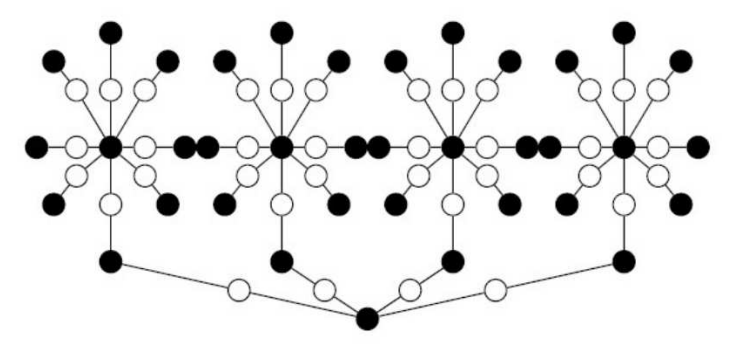

Figure 3: The subdivision of Banana Tree graph $B_{7,4}$.

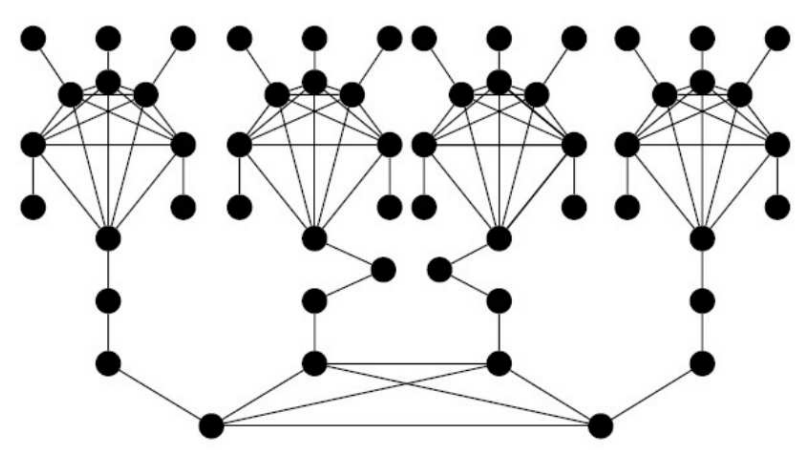

Figure 4: The line graph of subdivision of Banana Tree graph $B_{7,4}$. 
1. $M_{1}\left[L\left(S\left(B_{n, m}\right)\right)\right]=m(n-2) n+m(n+1)+m(m+2)+4 m$

$+m^{2}(m-1)+m(n-1)^{2}(n-2)$;

2. $M_{2}\left[L\left(S\left(B_{n, m}\right)\right)\right]=m(n-1)(n-2)+2 m(n-1)+2 m^{2}+4 m$ $+\frac{1}{2} m^{3}(m-1)+\frac{1}{2} m(n-1)^{3}(n-2)$;

3. $M_{3}\left[L\left(S\left(B_{n, m}\right)\right)\right]=-m(n-2)^{2}+m(3-n)+m(2-m)$;

4. $H M\left[L\left(S\left(B_{n, m}\right)\right)\right]=m n^{2}(n-2)+m(n+1)^{2}+m(m+2)^{2}$ $+16 m+2 m^{3}(m-1)+\frac{1}{2} m(n-1)(n-2)(2 n-2)^{2}$;

5. $A B C\left[L\left(S\left(B_{n, m}\right)\right)\right]=m(n-2) \sqrt{\frac{n-2}{n-1}}+\frac{3}{2} m \sqrt{2}+\frac{1}{2}(m-1) \sqrt{2 m-2}$ $+\frac{1}{2} m(n-2) \sqrt{2 n-4}$;

6. $R\left[L\left(S\left(B_{n, m}\right)\right)\right]=\frac{m(n-2)}{\sqrt{n-1}}+\frac{m}{\sqrt{2 n-2}}+\frac{1}{2} \sqrt{2 m}+\frac{1}{2} m+\frac{1}{2}(m-1)$ $+\frac{1}{2} m(n-2)$;

7. $G A\left[L\left(S\left(B_{n, m}\right)\right)\right]=2 m(n-2) \sqrt{\frac{n-1}{n}}+2 m \sqrt{\frac{2 n-2}{n+1}}+2 m^{\frac{3}{2}} \sqrt{\frac{2}{m+2}}$ $+m+\frac{1}{2} m(m-1)+\frac{1}{2} m(n-1)(n-2)$;

8. $\chi\left[L\left(S\left(B_{n, m}\right)\right)\right]=\frac{m(n-2)}{\sqrt{n}}+\frac{m}{\sqrt{n+1}}+\frac{m}{\sqrt{m+2}}+\frac{1}{2} m+\frac{1}{4} m(m-1) \sqrt{\frac{2}{m}}$ $+\frac{1}{2} \frac{m(n-1)(n-2)}{\sqrt{2 n-2}}$

9. $A Z I\left[L\left(S\left(B_{n, m}\right)\right)\right]=\frac{m(n-1)^{3}(n-2)}{(n-2)^{3}}+24 m+\frac{1}{2} \frac{m(m-1) m^{6}}{(2 m-2)^{3}}$ $+\frac{1}{2} \frac{m(n-1)^{7}(n-2)}{(2 n-4)^{3}}$.

Proof. The graph $L\left(S\left(B_{n, m}\right)\right)$ for $n=7$ and $m=4$ is shown in Fig. 4 . The order of $L\left(S\left(B_{n, m}\right)\right)$ is $2 m n$ out of which $m(n-2)$ vertices are of degree $1, m(n-1)$ vertices are of degree $n-1, m$ vertices are of degree $m$ and $2 m$ vertices are of degree 2. The size of $L\left(S\left(B_{n, m}\right)\right)$ is $\frac{m^{2}+3 m+m n^{2}-m n}{2}$. The edge partition of $L\left(S\left(B_{n, m}\right)\right)$ into the edges of type $\left(d_{u}, d_{v}\right)$ where $u v$ is an edge of $L\left(S\left(B_{n, m}\right)\right)$ is shown in Table 2 .

We obtain the required results by using formulas $(1)-(9)$.

Theorem 6. Let $L\left(S\left(B_{n, m}\right)\right)$ be the line graph of subdivision of Banana tree graph $B_{n, m}$. Then,

1. $M_{1}\left(\overline{\left[L\left(S\left(B_{n, m}\right)\right)\right]}\right)=m n(n-2)+m(n+1)+m(m+2)+4 m+m(m-$ $1)^{2}+m(n-1)(n-2)^{2}+2 m n(2 m n-1)^{2}-4 m n\left(m^{2}+3 m\right)+2$; 


\begin{tabular}{ccccccc}
\hline$\left(d_{u}, d_{v}\right)$ & $(1, n-1)$ & $(2, n-1)$ & $(2, m)$ & $(2,2)$ & $(m, m)$ & $(n-1, n-1)$ \\
\hline Number of edges & $m(n-2)$ & $m$ & $m$ & $m$ & $\frac{m(m-1)}{2}$ & $\frac{m(n-1)(n-2)}{2}$ \\
\hline
\end{tabular}

Table 2: The edge partition of $L\left(S\left(B_{n, m}\right)\right)$.

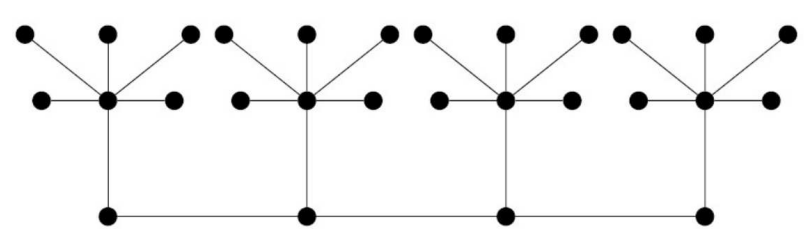

Figure 5: The Firecracker graph $F_{7,4}$.

2. $\overline{M_{1}}\left[L\left(S\left(B_{n, m}\right)\right)\right]=2 m n\left(m^{2}+3 m\right)-1-m n(n-2)-m(n+1)-m(m+$ 2) $-4 m-m(m-1)^{2}-m(n-1)(n-2)^{2}$;

3. $\overline{M_{2}}\left[L\left(S\left(B_{n, m}\right)\right)\right]=\frac{1}{2}\left(m^{2}+m n^{2}-m n+3 m\right)^{2}-\frac{1}{2} m n(n-2)-\frac{1}{2} m(n+$ 1) $-\frac{1}{2} m(m+2)-6 m-\frac{1}{2} m(m-1)^{2}-\frac{1}{2} m(n-1)(n-2)^{2}-2 m^{2} n^{2}(n-$ $\left.2)+m(n+1)+m(m+2)+4 m+m(m-1)^{2}+m(n-1)(n-2)^{2}\right)+\frac{3}{2}-$ $m(n-1)(n-2)-2 m(n-1)-2 m^{2}-\frac{1}{2} m(m-1)^{3}-\frac{1}{2} m(n-1)(n-2)^{3}$;

4. $\overline{M_{2}}\left(\overline{\left[L\left(S\left(B_{n, m}\right)\right)\right]}\right)=\frac{1}{2} m(2 m n-1)^{2}+m n(2 m n-1)^{2}-m n(2 m n-1)+$ $3 m(2 m n-1)^{2}-2 m n(m n(n-2))+m(n+1)+m(m+2)+40 m+m(m-$ $1)^{2}+m(n-1)(n-2)^{2}-65+4 n+3(m-2)(n-1)+\frac{1}{2} m(n-1)(n-2)^{3}+1+$ $m(n-1)(n-2)+2 m(n-1)+2 m^{2}+4 m+\frac{1}{2} m(m-1)^{3}+\frac{1}{2} m(n-1)(n-2)^{3}$.

Proof. We obtain the required results from Theorems 1, 2 and Table 2.

\section{Topological indices of line graph of subdivision of Firecracker} graph

The Firecracker graph $F_{n, m}$ is the graph obtained by the concatenation of $n m$-stars by linking one leaf from each. The subdivision of $F_{n, m}$ has order $(2 m n-1)$ and size $2(m n-1)$ as shown in the Fig. 6. 


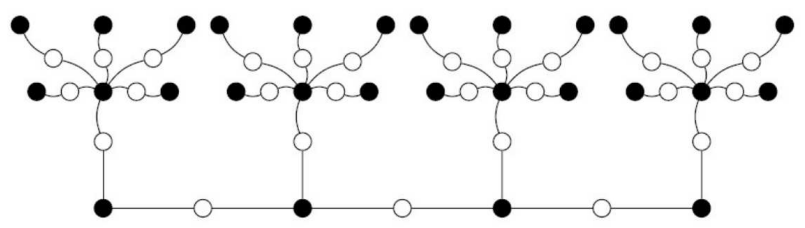

Figure 6: The subdivision of Firecracker graph $F_{7,4}$.

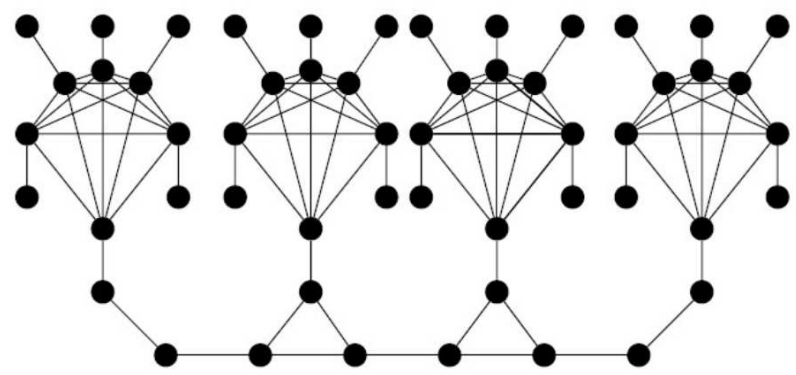

Figure 7: The line graph of subdivision of Firecracker graph $F_{7,4}$.

Theorem 7. Let $\left[L\left(S\left(F_{n, m}\right)\right)\right]$ be the subdivision of line graph of the Firecracker graph $F_{n, m}$. Then

1. $M_{1}\left[L\left(S\left(F_{n, m}\right)\right)\right]=24 m+2 n+(m-2)(n+2)+m(n-1)^{2}(n-2)-34$;

2. $M_{2}\left[L\left(S\left(F_{n, m}\right)\right)\right]=36 m+4 n+3(m-2)(n-1)+m(n-1)^{2}(n-2)-65$;

3. $M_{3}\left[L\left(S\left(F_{n, m}\right)\right)\right]=4-2 n-(m-2)(n-4)$;

4. $H M\left[L\left(S\left(F_{n, m}\right)\right)\right]=144 m+2(n+1)^{2}+(m-2)(n+2)^{2}+2 m(n-1)^{3}(n-$ 2) - 242;

5. $A B C\left[L\left(S\left(F_{n, m}\right)\right)\right]=3 \sqrt{2}+\frac{8}{3} m-6+\frac{1}{3}(m-2) \sqrt{\frac{3 n}{n-1}}+\frac{1}{2} m(n-2) \sqrt{2 n-4}$;

6. $R\left[L\left(S\left(F_{n, m}\right)\right)\right]=\frac{1}{3} \sqrt{6}+\frac{4}{3} m+\frac{2}{\sqrt{2 n-2}}+\frac{m-2}{\sqrt{3 n-3}}+\frac{1}{2} \frac{m(n-1)(n-2)}{\sqrt{(n-1)^{2}-2}}$;

7. $G A\left[L\left(S\left(F_{n, m}\right)\right)\right]=-7+\frac{4 \sqrt{6}}{5}+4 m+\frac{\sqrt{2 n-2}(1+n)}{+} \frac{2(m-2) \sqrt{3 n-3)}}{(2+n)}+\frac{m(n-1)^{2}(n-2)}{(2 n-2)}$;

8. $\chi\left[L\left(S\left(F_{n, m}\right)\right)\right]=1+\frac{2}{\sqrt{5}}+\frac{1}{\sqrt{6}}(4 m-9)+\frac{2}{\sqrt{1+n}}+\frac{(m-2)}{\sqrt{2+n}}+\frac{1}{2} \frac{m(n-1)(n-2)}{\sqrt{2 n-2}}$; 


\begin{tabular}{ccccccc}
\hline$\left(d_{u}, d_{v}\right)$ & $(2,2)$ & $(2,3)$ & $(3,3)$ & $(2, n-1)$ & $(3, n-1)$ & $(n-1, n-1)$ \\
\hline Number of edges & 2 & 2 & $4 m-9$ & 2 & $m-2$ & $\frac{m(n-1)(n-2)}{2}$ \\
\hline
\end{tabular}

Table 3: The edge partition of $L\left(S\left(F_{n, m}\right)\right)$.

9. $A Z I\left[L\left(S\left(F_{n, m}\right)\right)\right]=\frac{-3489}{64}+\frac{729}{16} m+\frac{27(m-2)(n-1)^{3}}{n^{3}}+\frac{1}{2} \frac{m(n-1)^{7}(n-2)^{3}}{(2 n-4)}$.

Proof. The graph $L\left(S\left(F_{n, m}\right)\right)$ for $m=4$ and $n=7$ is shown in Fig. 7 . The order of $L\left(S\left(F_{n, m}\right)\right)$ is $2(m n-1)$ out of which $m(n-2)$ vertices are of degree 1,4 vertices are of degree $2, m(n-1)$ vertices are of degree $n-1,3(m-2)$ vertices are of degree 3 . The size of $L\left(S\left(F_{n, m}\right)\right)$ is $\frac{m n^{2}-m n+8 m-10}{2}$. The edge partition of $L\left(S\left(F_{n, m}\right)\right)$ into edges of the type $\left(d_{u}, d_{v}\right)$ where $u v$ is an edge of $L\left(S\left(F_{n, m}\right)\right)$ is shown in Table 3 .

We obtain the required results by using formulas $(1)-9$ ).

Theorem 8. Let $L\left(S\left(F_{n, m}\right)\right)$ be the line graph of subdivision of the Firecracker graph $F_{n, m}$. Then

1. $M_{1} \overline{\left[L\left(S\left(F_{n, m}\right)\right)\right]}=24 m+2 n+(m-2)(n+2)+m(n-1)^{2}(n-2)+$ $2(m n(2 m n-3)-1)^{2}-2 m n(2 m n-3)^{2}+2 m n(2 m n-3)-16 m(2 m n-3)-14$;

2. $\overline{M_{1}}\left[L\left(S\left(F_{n, m}\right)\right)\right]=\overline{M_{1}}\left(\overline{\left[L\left(S\left(F_{n, m}\right)\right)\right]}\right)=2 m n(2 m n-3)^{2}-2 m n(2 m n-$ $3)+16 m(2 m n-3)+14+24 m+2 n+(m-2)(n+2)+m(n-1)^{2}(n-2)$;

3. $M_{2} \overline{\left[L\left(S\left(F_{n, m}\right)\right)\right]}=(m n(2 m n-3)-1)^{3}-3\left(\frac{1}{2} m n^{2}-\frac{1}{2} m n+4 m-5\right)(2 m n-$ $3)^{2}+\frac{1}{2} m n^{2}-\frac{1}{2} m n+40 m+\frac{113}{2}+2 m n(-34+24 m+2 n)+(m-2)(n+$ $2)+\left(m(n-1)^{2}(n-2)\right)+4 n+3(m-2)(n-1)+\frac{1}{2} m(n-1)^{3}(n-2)$;

4. $\overline{M_{2}}\left[L\left(S\left(F_{n, m}\right)\right)\right]=\frac{1}{2} m n^{2}-\frac{1}{2} m n+28 m+77+3 n-\frac{1}{2}(m-2)(n+2)-$ $\frac{1}{2} m(n-1)^{2}(n-2)+3(m-2)(n-1)+\frac{1}{2} m(n-1)^{3}(n-2)$;

5. $\left.\overline{M_{2}} \overline{\left[L\left(S\left(F_{n, m}\right)\right)\right]}=\frac{1}{2} m n(2 m n-3)^{2}-m n(2 m n-3)+8 m(2 m n-3)-10\right)^{2}-$ $2 m n\left(-34+24 m+2 n+(m-2)(n+2)+m(n-1)(n-2)^{2}-62+36 m+\right.$ $4 n+3(m-2)(n-1)+\frac{1}{2} m(n-1)(n-2)^{3}$.

Proof. We obtain the required results from Theorems 1, 2 and Table 3. 


\section{Acknowledgments}

This work is supported by the Funding: Hefei University Annual Academy Research Development Fund Project (Natural Science) No. 18ZR12ZDA.

\section{References}

[1] M.O. Alberton, The irregularity of a graph, Ars. Combin., 46 (1997), 219225.

[2] K. Ali, E.T. Baskoro, I. Tomescu, On the Ramzey number of paths and Jahangir graph $J_{3, m}$, Bull. Math. Soc. Sci. Math. Roumanie, Tome 51(99), No 3 (2008), 177-182.

[3] F. Asif, Z. Zahid, S. Zafar, M. R. Farahani, W. Gao, On topological properties of some convex polytopes by using line operator on their subdivisions, Hacet. J. Math. Stat., (2019), 1-10; DOI: 10.15672/HJMS.2019.671.

[4] S.H. Bertz, The bond graph, J. C. S. Chem. Commun., (1981), 818-820.

[5] K.Ch. Das, K. Das, I. Gutman, Some properties of the second Zagreb index, MATCH Commun. Math. Comput. Chem., 52 (2004), 103-112.

[6] E. Estrada, L. Torres, L. Rodriguez, I. Gutman, An atom-bond connectivity index: Modelling the enthalpy of formation of alkanes, Indian J. Chem., 37 (1998), 849-855.

[7] G.H. Fath-Tabar, Old and new Zagreb indices of graphs, MATCH Commun. Math. Comput. Chem., 65 (2011), 79-84.

[8] M.R. Farahani, M.F. Nadeem, S. Zafar, Z. Zahid, M.N. Husin, Study of the topological indices of the line graphs of H-Pantacenic nanotubes, New Front. Chem., 26, No 1 (2017), 31-38.

[9] Z. Foruzanfar, F. Asif, Z. Zahid, S. Zafar, M. R. Farahani, $A B C_{4}$ and $G A_{5}$ indices of line graph of subdivision of some convex polytopes, Indian $J$. Pure Appl. Mat., 117, No 4 (2017), 645-653.

[10] Z. Foruzanfar, F. Asif, Z. Zahid, S. Zafar, M.R. Farahani, $A B C_{4}$ and $G A_{5}$ indices of para-line graph of some convex polytopes, Stat., Optim. Inf. Comput., 7 (2019), 192-197. 
[11] B. Furtula, A. Graovac, D. Vukicevic, Augmented Zagreb index, J. Math. Chem., 48 (2010), 370-380.

[12] I. Gutman, Selected properties of the schultz molecular topological index, J. Chem. Inf. Comput. Sci., 34 (1994), 1087-1089.

[13] I. Gutman, K.C. Das, The first Zagreb index 30 years after, MATCH Commun. Math. Comput. Chem., 50 (2004), 83-92.

[14] I. Gutman, B. Furtula, Z.K. Vukićević, G. Popivoda, On Zagreb indices and coindices, MATCH Commun. Math. Comput. Chem., 74 (2015), 5-16.

[15] I. Gutman, B. Furtula, A.A. Toropov, A.P. Toropova, The graph of atomic orbitals and its basic properties. 2. Zagreb indices, MATCH Commun. Math. Comput. Chem., 53 (2005), 225-230.

[16] I. Gutman, L. Popovic, B.K. Mishra, M. Kaunar, E. Estrada, N. Guevara, Application of line graphs in physical chemistry. Predicting surface tension of alkanes, J. Serb. Chem. Soc., 62 (1997), 1025-1029.

[17] I. Gutman, N. Trinajstić, Graph theory and molecular orbitals. Total $\pi$ electron energy of alternant hydrocarbons, Chem. Phys. Lett., 17 (1972), $535-538$.

[18] I. Gutman, Z. Tomovic, On the application of line graphs in quantitative structure-property studies, J. Serb. Chem. Soc., 65, No 8 (2000), 577-580.

[19] P. Hansen, H. Melot, I. Gutman, Variable neighborhood search for extremal graphs 12. A note on the variance of bounded degrees in graphs, MATCH Commun. Math. Comput. Chem., 54 (2005), 221-232.

[20] Y. Huo, J.B. Liu, Z. Zahid, S. Zafar, M.R. Farahani, M.F. Nadeem, On certain topological indices of the line graph of $\mathrm{CNCk}[\mathrm{n}]$ nanocones, J. Comput. Theor. Nanosci., 13 (2016), 4318-4322.

[21] M.N. Husin, F. Asif, Z. Zahid, S. Zafar, M.R. Farahani, On topological properties of plane graphs by using line operator on their subdivisions, Adv. Appl. Disc. Math., 19, No 4 (2018), 479-490.

[22] A. Iranmanesh, I. Gutman, O. Khormali, A. Mahmiani, The edge versions of the Wiener index, MATCH Comm. Math. Comput. Chem., 61 (2009), 663-672. 
[23] M.F. Nadeem, S. Zafar, Z. Zahid, On topological properties of the line graphs of subdivision graphs of certain nanostructures, Appl. Math. Comput., 273 (2016), 125-130.

[24] M.F. Nadeem, S. Zafar, Z. Zahid, Some topological indices of $L\left(S\left(C N C_{k}[n]\right)\right.$, Punjab Univ. J. Math., 49, No 1 (2017), 13-17.

[25] M.F. Nadeem, S. Zafar, Z. Zahid, On the edge version of geometricarithmetic index of nanocones, Stud. U. Babes-Bol. Chem., 61, No 1 (2016), 273-282.

[26] M. Randic, On characterization of molecular branching, J. Amer. Chem. Soc., 97 (1975), 6609-6615.

[27] G.H. Shirdel, H. Rezapour, A.M. Sayadi, The hyper-Zagreb index of graph operations, Iran. J. Math. Chem., 4, No 2 (2013), 213-220.

[28] D. Vukicevic, B. Furtula, Topological index based on the ratios of geometrical and arithmetical means of end-vertex degrees of edges, J. Math. Chem., 46 (2009), 1369-1376.

[29] B. Zhou, N. Trinajstic, On general sum-connectivity index, J. Math. Chem., 47 (2010), 210-218.

[30] B. Zhou, Zagreb indices, MATCH Commun. Math. Comput. Chem., 52 (2004), 113-118. 